\title{
Administration of crizotinib via jejunostomy tube: A case report
}

\author{
Lotte M Knapen 1 , Anne-Marie C Dingemans², Sander Croes ${ }^{{ }^{*}}$
}

\author{
${ }^{*}$ Correspondence \\ S Croes \\ Department of Clinical Pharmacy and \\ Toxicology, CAPHRI - Care and Primary \\ Health Research Institute, Maastricht \\ University Medical Center (MUMC+), \\ Maastricht, The Netherlands. \\ Email: s.croes@mumc.nl \\ Telephone number: +31-43-3871431 \\ 1Department of Clinical Pharmacy and \\ Toxicology, CAPHRI - Care and Primary \\ Health Research Institute, Maastricht \\ University Medical Center (MUMC+), \\ Maastricht, The Netherlands. \\ 2Department of Pulmonary Diseases, \\ GROW - School for Oncology and \\ Developmental Biology, Maastricht \\ University Medical Center (MUMC+), \\ Maastricht, The Netherlands. \\ Received: Jun 12, 2018 \\ Accepted: Jul 18, 2018 \\ Published: Jul 25, 2018 \\ (C) Lotte M Knapen et al, 2018; licensee \\ OA Journal of Case Reports. This is an \\ Open Access article distributed under \\ the terms of the Creative Commons \\ Attribution License (http:// \\ creativecommons.org/licenses/by/4.0)
}

\begin{abstract}
Crizotinib is an orally available tyrosine kinase inhibitor, approved for treatment of anaplastic lymphoma kinase (ALK) and c-ros oncogene 1 (ROS1) rearrangement-positive non-small cell lung cancer (NSCLC). According to the product leaflet, crizotinib capsules should be swallowed whole, and should not be crushed, dissolved or opened. However, this manner of administration is not always possible. At present, literature is lacking regarding the absorption of crizotinib via percutaneous endoscopic jejunostomy (PEJ) tube. We report a case of a patient with ALK+ NSCLC who was administered crizotinib via PEJ tube. An adequate steady state crizotinib trough concentration was reached, resulting in a metabolic response. Safety for the caregiver was ensured since the administration of crizotinib was made without crushing or opening the capsule. This case supports the option for providing crizotinib via PEJ tube in patients who have ALK+ NSCLC and are unable to swallow whole capsules. This option might also apply to the administration of other ALK inhibitors.
\end{abstract}

Keywords: Crizotinib, ALK inhibitor, percutaneous endoscopic jejunostomy tube, pharmacokinetics, non-small cell lung cancer.

Abbreviations: ALK: anaplastic lymphoma kinase, HPLC-MS/MS: highperformance liquid chromatography-tandem mass spectrometry, NG: nasogastric, NKI-AVL: Netherlands Cancer Institute-Antoni van Leeuwenhoek, NSCLC: non-small cell lung cancer, PEG: percutaneous endoscopic gastronomy, PE): percutaneous endoscopic jejunostomy, PET-CT: positron emission tomography-computed tomography, PVC: polyvinyl chloride, ROS1: c-ros oncogene 1.

\section{Introduction}

Crizotinib is an orally available small-molecule inhibitor of multiple tyrosine kinases, and approved for treatment of anaplastic lymphoma kinase (ALK) and c-ros oncogene 1 (ROS1) rearrangement-positive non-small cell lung cancer (NSCLC) [1]. Crizotinib is administered as a capsule of $250 \mathrm{mg}$ taken orally twice daily. The capsules may be taken with or without food and should not be crushed, dissolved or opened [1]. The oral administration is an advantage. However, it is not always possible to administer crizotinib orally as whole capsules. It is unknown whether alternative routes of administration for patients who cannot take the drug orally leads to adequate exposure of crizotinib. At present, literature is lacking regarding the absorption of crizotinib via percutaneous endoscopic jejunostomy (PEJ) tube. There is only one case report in which crizotinib was administrated via nasogastric (NG) and percutaneous endoscopic gastrostomy (PEG) tubes. However, no serum crizotinib concentrations were measured to demonstrate adequate exposure of crizotinib when applying these methods of administration [2]. 
To our knowledge, this is the first report of a patient having achieved an adequate steady state crizotinib trough concentration after receiving crizotinib administered via PEJ tube.

\section{Case Presentation}

A 36-year-old female patient, known with ALK+ translocated NSCLC, presented in 2016 with recurrent disease with distant metastasis after previous chemoradiation and resection. The preferred treatment was crizotinib. However, due to complications of the previous treatment, oral intake was not possible and she received nutrition by PEJ. Crizotinib was started at a dose of $250 \mathrm{mg}$ twice daily, which was administered via PEJ tube as a dispersion in lukewarm water. The method of administration was similar as being described by Tamai et al [2]. Since the specific site of absorption of crizotinib within the gastrointestinal system is currently unknown, it was decided to determine a steady state crizotinib trough concentration at day 20 of therapy [1]. The crizotinib trough concentration was analyzed by a validated high-performance liquid chromatography-tandem mass spectrometry (HPLCMS/MS) method in a laboratory of the Netherlands Cancer Institute-Antoni van Leeuwenhoek (NKI-AVL). The crizotinib trough concentration reached was 304 $\mathrm{ng} / \mathrm{mL}$. Therapy was continued at a dose of $250 \mathrm{mg}$ twice daily. No tube obstruction or adverse events were seen. Tumour response was radiographic assessed by positron emission tomographycomputed tomography (PET-CT) according to RECIST version 1.0 and revealed a metabolic response after two months of treatment.

\section{Discussion}

The steady state crizotinib trough concentration reached in this patient, $304 \mathrm{ng} / \mathrm{mL}$, was in line with crizotinib concentrations described in literature, ranging from respectively 244 to $848 \mathrm{ng} / \mathrm{mL}$ in Asian populations [3] and 242 to $319 \mathrm{ng} / \mathrm{mL}$ in Caucasian populations [4].

We had access to the electronic patient record and the pharmacy dispensing data which contained large amount of information regarding multiple factors known to influence the exposure of crizotinib. First of all, we performed a medication review since crizotinib is known to be a CYP3A4 substrate, a moderate time-dependent inhibitor, and a weak inducer [ $\underline{5}$. We found no drug-drug interactions. However, our patient was using pantoprazole $40 \mathrm{mg}$ once daily, which was administered via PEJ tube $[\underline{1}, \underline{6}]$. The jejunal $\mathrm{pH}$ is significantly higher than the gastric $\mathrm{pH}$ and the aqueous solubility of crizotinib is $\mathrm{pH}$ dependent, with low (acidic) pH resulting in higher solubility [1, 7]. However, the exposure of crizotinib was not affected in a significant manner by the use of pantoprazole and the administration via jejunal tube. This result is in line with literature [ $\left[\begin{array}{ll}1 & 6\end{array}\right]$. Furthermore, since our patient was fed by enteral nutrition, the nutritional composition over time was stable. Although the effect of food on steady state crizotinib exposure is currently unknown, it is described that a high-fat meal decreases the systemic exposure by an average of $15 \%$ compared to fasting conditions [1]. Moreover, the patient did not use any food (e.g. grapefruit juice) or herbal supplements (e.g. St. John's wort). The pharmacokinetic covariates of the patient (Caucasian female, 36 years, $\pm 55 \mathrm{~kg}$, non-smoker, normal renal and hepatic function) were not expected to significantly influence the exposure of crizotinib [8].

A multitude of factors need to be accounted for when planning to deliver drugs by feeding tubes. Product alterations necessary to allow tube delivery may interfere with product stability, compatibility with concomitant medications and tolerability, as well as pharmacokinetic parameters [9]. However, crizotinib is an immediate-release formulated capsule and can be administered by any tube comprised of polyurethane, polyvinyl chloride (PVC) or silicone $[1,10]$. The crizotinib administration was performed via dissolution of the formulated capsule into a $20 \mathrm{~mL}$ syringe containing lukewarm water [10]. No specific safety precautions were necessary since the capsule did not have to be crushed or opened [10]. However, it is important for caregivers, who handle the medication, to avoid skin contact with crizotinib. Wearing disposable gloves should be advised in order to safely transfer the capsule from the package to the syringe.

It is possible to assume that the described administration method can also be applied for second and third-generation ALK inhibitors, candidates being alectinib, brigatinib, ceritinib and lorlatinib [11]. Similar to other tyrosine kinase inhibitors, these agents have been developed as 
immediate-release formulated capsules and tablets, and not as delayed-, extended-, or sustained-release formulations [12-14]. In addition, all agents are not enteric coated. The instruction to take capsules and tablets without crushing, dissolving or opening seems to be provided to minimize environmental exposure of these agents. Adequate absorption cannot be guaranteed by administrating these agents as dispersions via NG, PEG or PEJ enteric feeding tubes. Similar to our report, concentration measurements during steady state may be performed to determine exposure. However, this is only possible when validated analytical methods are available. Future pharmacokinetic studies are warranted to investigate these methods of administration.

\section{Conclusion}

In this case report, we demonstrate adequate crizotinib absorption after administration via PEJ tube with preservation of the steady state trough crizotinib concentration. This method of administration might be applicable to other ALK inhibitors.

\section{Acknowledgement}

None.

\section{Funding}

Not applicable.

\section{Conflicts of Interest}

Lotte M Knapen: No relationships to disclose.

Anne-Marie C Dingemans: Advisory boards: Roche, Eli Lilly, Boehringer Ingelheim, Astra Zeneca, Pfizer, BMS, Amgen, Novartis, MSD.

Sander Croes: No relationships to disclose.

\section{References}

1. Xalkori (2018) Summary of Product Characteristics.

2. Tamai K, Nagata K, Otsuka K, et al. (2013) Crizotinib administered via nasogastric and percutaneous endoscopic gastrostomy tubes for the successful treatment of ALKrearranged lung cancer in a patient with poor performance status. Respir Investig. 51: 46-48.

3. Kurata Y, Miyauchi N, Suno M, et al. (2015) Correlation of plasma crizotinib trough concentration with adverse events in patients with anaplastic lymphoma kinase positive non-smallcell lung cancer. J Pharm HealthCare Sci. 1: 8.

4. CDER (2011) Application Number: 2025700rig1s000, Clinical Pharmacology and Biopharmaceutics Review(s).

5. Yamazaki S, Johnson TR, Smith BJ (2015) Prediction of DrugDrug Interactions with Crizotinib as the CYP3A Substrate Using a Physiologically Based Pharmacokinetic Model. Drug Metab Dispos. 43: 1417-1429.

6. Smelick GS, Heffron TP, Chu L, et al. (2013) Prevalence of acidreducing agents (ARA) in cancer populations and ARA drugdrug interactions potential for molecular targeted agents in clinical development. Mol Pharm. 10: 4055-4062.

7. Lindahl A, Ungell AL, Knutson L, et al. (1997) Characterization of fluids from the stomach and proximal jejunum in men and women. Pharm Res. 14: 497-502.

8. Wang E, Nickens DJ, Bello A, et al. (2016) Clinical Implications of the Pharmacokinetics of Crizotinib in Populations of Patients with Non-Small Cell Lung Cancer. Clin Cancer Res. 22: 5722-5728.

9. Stott KE, Singh B, Beadsworth MB, et al. (2016) Adequacy of Rifampin Absorption after Jejunostomy Tube Administration. Pharmacotherapy. 36: e23-e25.

10. Xalkori (2016) MedInfo Letter Pfizer.

11. Faccinetti F, Tiseo M, Maio M, et al. (2016) Tackling ALK in nonsmall cell lung cancer: the role of novel inhibitors. Transl Lung Cancer Res. 5: 301-321.

12. Alectinib (2018) Summary of Product Characteristics, FDA.

13. Ceritinib (2018) Summary of Product Characteristics, FDA.

14. Brigatinib - Clinical Investigator Brochure. ARIAD Pharmaceuticals, Incorporation, AP26113. 